\title{
Effects of Satureja khuzestanica on Serum Glucose, Lipids and Markers of Oxidative Stress in Patients with Type 2 Diabetes Mellitus: A Double-Blind Randomized Controlled Trial
}

\author{
Sanaz Vosough-Ghanbari ${ }^{1}$, Roja Rahimi ${ }^{1}$, Shabnam Kharabaf ${ }^{1}$, Shima Zeinali ${ }^{1}$, \\ Azadeh Mohammadirad ${ }^{1}$, Somayeh Amini $^{2}$, Nargues Yasa ${ }^{3}$, Alinazar Salehnia ${ }^{1}$, \\ Tayebeh Toliat ${ }^{1}$, Shekoufeh Nikfar ${ }^{4}$, Bagher Larijani ${ }^{2}$ and Mohammad Abdollahi ${ }^{1}$
}

${ }^{1}$ Faculty of Pharmacy and Pharmaceutical Sciences Research Center (PSRC), ${ }^{2}$ Endocrinology and Metabolism Research Center (EMRC) and Faculty of Medicine, ${ }^{3}$ Medicinal Plants Research Center (MPRC), Tehran University of Medical Sciences (TUMS) and ${ }^{4}$ Drug Selecting Committee, Food and Drug Organization, and Food and Drug Laboratory Research Center, Tehran, Iran

\begin{abstract}
Satureja khuzestanica is an endemic plant of Iran that is widely distributed in the Southern part of the country. It has antioxidant properties and thus it seems to be useful in diseases related to oxidative stress such as diabetes and hyperlipidemia. The present study investigates the effect of $S$. khuzestanica supplement in metabolic parameters of hyperlipidemic patients with type 2 diabetes mellitus. Twenty-one hyperlipidemic patients with type 2 diabetes mellitus were randomized in a double blind, placebo controlled clinical trial to receive either S. khuzestanica (tablets contain $250 \mathrm{mg}$ dried leaves) or placebo once a day for 60 days. Blood samples were obtained at baseline and at the end of the study. Samples were analyzed for levels of glucose, total cholesterol, LDL-cholesterol, HDL-cholesterol, triglyceride, creatinine, thiobarbituric acid reactive substances (TBARS) as marker of lipid peroxidation and ferric reducing ability (total antioxidant power, TAP). Treatment of patients by $S$. khuzestanica for 60 days induced significant decrease in total cholesterol $(P=0.008)$ and LDL-cholesterol $(P=0.03)$ while increased HDL-cholesterol $(P=0.02)$ and TAP $(P=0.007)$ in comparison with the baseline values. S. khuzestanica did not alter blood glucose, triglyceride, creatinin and TBARS levels. In comparison with baseline values, no significant change was observed in blood glucose, total cholesterol, LDL-cholesterol, HDL-cholesterol, triglyceride, creatinine, TBARS and TAP in placebo-treated group. Usage of S. khuzestanica as a supplement to drug regimen of diabetic type 2 patients with hyperlipidemia is recommended.
\end{abstract}

Keywords: hyperlipidemia - oxidative stress - Satureja khuzestanica - type 2 diabetes mellitus

\section{Introduction}

Satureja khuzestanica Jamzad (Marzeh khuzestani in Persian, family of Lamiaceae, sub family of Nepetoidae)

For reprints and all correspondence: Prof. Mohammad Abdollahi, Laboratory of Toxicology, Faculty of Pharmacy, and Pharmaceutical Sciences Research Center, Tehran University of Medical Sciences, Tehran, PO Box 14155-6451, Iran. Tel: +98-21-66959104;

Fax: +98-21-66959104; E-mail: mohammad@tums.ac.ir is an endemic plant that is widely distributed in the Southern parts of Iran. It is a subshrub, branched stem $\sim 30 \mathrm{~cm}$ high, densely leafy, broadly ovaiate-orbicular covered with white hairs (1). Base of the leaves is attenuate and petioliform. It is famous for its therapeutic value as an analgesic and antiseptic in folk medicine (2).

In the recent years, antimicrobial (3-5), antiviral $(6,7)$, antioxidant (8-10), antiproliferative (11), antiprotozoal 
$(12,13)$, antifungal $(14,15)$, anti-inflammatory and antinociceptive $(16,17)$, antidiarrheal (18) and vasodilatory $(19,20)$ activities were reported from different species of Satureja.

It has been shown that hydroethanolic extract of S. khuzestanica has anti-inflammatory and antinociceptive properties in carrageenan-induced rat paw edema and formalin test comparable with those of indomethacin and morphine (21). The methanolic extract of the aerial parts of $S$. khuzestanica has been investigated for its antimicrobial activity. The maximum antibacterial and antifungal activities has been observed against Staphylococcus aureus and Candida albicans (22). Significant decrease in fasting blood glucose and triglyceride levels were observed with essential oil of $S$. khuzestanica in diabetic and hyperlipidemic rats. A significant decrease in the normal lipid peroxidation and an increase in body antioxidant power were reported while the cholesterol level did not change significantly in hyperlipidemic rats (23). It has been reported that essential oil from $S$. khuzestanica does not affect blood glucose concentrations but decreases hepatic phosphoenolpyruvate carboxy kinase activity and increases hepatic glycogen phosphorylase in rats (24). Study on the effect of oral administration of essential oil from S. khuzestanica in male rat fertility has revealed significant improvement in all parameters of libido such as potency, fecundity, fertility index and litter size. Moreover, concentrations of FSH and testosterone and the weights of testes, seminal vesicles and ventral prostate weights were significantly increased. Histopathological analysis showed that the number of spermatogonium, spermatid cords, Leydig cells and spermatozoids was increased and Sertoli cells were hypertrophic (25). Essential oil from $S$. khuzestanica has protected mice from experimentally induced inflammatory bowel disease and significantly decreased lipid peroxidation in bowel of animals (26). Primary acute toxicity and teratogenicity tests were also performed and confirmed plant's safety (23).

One of the specific identifying characteristics of the subfamily Nepetoidae is that its representatives contain $<0.5 \%$ essential oil (27). The main component of essential oil of S. khuzestanica is carvacrol (28). Other constituents identified in this plant are flavones, triterpenoids, steroids and tannins (29). Both carvacrol and flavonoids have been found to have antioxidant properties (30-33). Lipid peroxidation and consequent oxidative stress are increased in diabetes and hyperlipidemia, and have been proposed to play roles in the pathogenesis of these diseases (34-37). The above-mentioned studies in animals confirmed the safety of S. khuzestanica. In the present study, the antidiabetic, antihyperlipidemic and antioxidant effects of $S$. khuzestanica were investigated in patients with type 2 diabetes mellitus.

\section{Methods}

\section{Plant Collection and Tablet Preparation}

The aerial parts of the S. khuzestanica were collected during the flowering stage in June 2000 from Khorramabad in the Lorestan province of Iran. The plant was identified by the Department of Botany of the Research Institute of Forests and Rangelands (TARI) in Tehran. A voucher specimen (No. 58416) has been deposited at the TARI Herbarium. The plant was cultivated in Khorramabad and the aerial parts of the plant were collected during the flowering stage. The aerial parts were air dried at ambient temperature in the shade. Tablets were prepared from dried leaves of plant (each tablet contained $250 \mathrm{mg}$ of dried leaves). Other ingredients of tablet were lactose, microcrystalline cellulose, starch, poly vinyl pyrollidone (PVP) and magnesium estearate. These ingredients were pressed with hardness of $5-7 \mathrm{~kg}$ and the final mean weight of tablet was $375 \mathrm{mg}$. The pressed tablets were film coated with hydroxy propyl methyl cellulose, poly ethylene glycol 4000, talc, titanium dioxide, PVP, distilled water and ethanol.

\section{Patient Selection}

In this randomized, prospective, double-blind controlled clinical trial, the database contains details of patients' age, sex, clinical history, previous pharmacologic therapies, hospitalizations and specialist consultations. We recruited 21 (12 male and 9 female) patients with type 2 diabetes mellitus with a mean age of 50.65 from outpatient clinic of a University Hospital. Diabetic patients were chosen according to American Diabetes Association (ADA) criteria (38). The patients were controlled by diet and oral antidiabetic drugs. Diabetics with hyperlipidemia who met the criteria for pharmacologic treatment according to National Cholesterol Education Program (NCEP) Adult Treatment Panel III (ATP III) guideline for diabetic patients were recruited (39). All patients were receiving antidiabetic and antihyperlipidemic medications at least 3 months before beginning the experiment.

Exclusion criteria were the followings: uncontrolled hypertension (systolic blood pressure $>140 \mathrm{~mm} \mathrm{Hg}$ and/ or diastolic blood pressure $>90 \mathrm{~mm} \mathrm{Hg}$ ); uncontrolled hypothyroidism; obstructive hepatic or biliary disease; alcoholism, smoking; autoimmune disease; chronic pancreatitis; active liver disease or hepatic dysfunction [i.e. alanine aminotransferase (ALT), aspartate aminotransferase (AST) and total bilirubin higher than 2-fold the upper limit of normal]; nephritic syndrome or renal dysfunction (i.e. serum creatinine concentration $>1.5 \mathrm{mg} \mathrm{dl}^{-1}$ ); concomitant participation in other clinical studies; use of heparin and/or oral anticoagulants; pulmonary infections; gangrene; diabetic foot ulceration; treatment with insulin and/or use of multivitamins and 
herbal traditional supplements in last 3 months. Patients treated with steroids (unless administered topically or for post-menopausal hormone replacement therapy), cyclosporine A, erythromycin, or ketoconazole were also excluded; as well as patients with a history of myocardial infarction, angioplasty, or major surgery in the 6 months before the beginning of the study. Pregnant, possibly pregnant, or breastfeeding women were excluded from the study. Women of childbearing age were required to use an effective method of birth control throughout the study.

The subjects were completely informed of the purpose, procedure and hazards of the trial and were free to leave the trial at any time. All patients signed informed written consent before being included in the study. The research followed guidelines of the Declaration of Helsinki and Tokyo for humans, and was approved by Ethics Committee on Human Experimentation of PSRC/TUMS.

Patients who met the eligibility criteria were randomly assigned to one of the two treatment groups according to a simple randomization scheme. Group I $(n=11)$ received $S$. khuzestanica tablets $(250 \mathrm{mg})$ once a day for 60 days and group II $(n=10)$ received placebo with the same regimen. The subjects were asked not to alter their usual diets and physical activity throughout the study and any changes in their medication were avoided whenever possible.

\section{Sample Collection and Handling}

After $12-14 \mathrm{~h}$ overnight fasting, between 08:00 and $10: 30 \mathrm{~h}$ and before taking any oral hypoglycemic agent(s), $10 \mathrm{ml}$ blood sample was collected from each subject in tubes containing heparin, before beginning of experiment and after 60 days administration of S. khuzestanica or placebo. After centrifugation of blood at $3000 \times g$ for $30 \mathrm{~min}$ at $4^{\circ} \mathrm{C}$, the plasma supernatant fluid was separated and stored at $-80^{\circ} \mathrm{C}$ until analyzed further.

Body mass index, blood pressure, serum total cholesterol (TC), low-density lipoprotein-cholesterol (LDL-C), high-density lipoprotein-cholesterol (HDL-C), triglyceride (TG), fasting blood glucose and serum creatinine were measured at the start and after 60 days of treatment.

\section{Total Antioxidant Power (TAP) Assay}

Antioxidant power of plasma was determined by measuring their ability to reduce $\mathrm{Fe}^{3+}$ to $\mathrm{Fe}^{2+}$ established as named (ferric reducing ability of plasma, FRAP) test that described previously (40). Briefly, in this test, the medium is exposed to $\mathrm{Fe}^{3+}$ and the antioxidants present in medium start to produce $\mathrm{Fe}^{2+}$ as an antioxidant activity. The FRAP reagent prepared freshly, contained $25 \mathrm{ml}$ of $300 \mathrm{mM}$ acetate buffer $(\mathrm{pH} 3.6)$ plus $2.5 \mathrm{ml}$ of $10 \mathrm{mM}$ 2,4,6-tripyridyl-s-triazine (TPTZ) solution in
$40 \mathrm{mM} \mathrm{HCl}$ and $2.5 \mathrm{ml}$ of $20 \mathrm{mM}$ ferric chloride $\left(\mathrm{FeCl}_{3}-6 \mathrm{H} 2 \mathrm{O}\right)$. The complex between $\mathrm{Fe}^{2+}$ and TPTZ gives a blue color with absorbance at $593 \mathrm{~nm}$.

\section{Lipid Peroxidation Assay}

The method based on the reaction of malonedialdehyde (MDA) as the end product of the oxidation of polyunsaturated fatty acids and its concentration in the medium is an established measure of lipid peroxidation extent. In this test the reaction of MDA with TBA creates a complex which is determined spectrophotometrically while lipid peroxidation in samples are assessed in terms of thiobarbituric acid (TBA) thiobarbituric acid reactive substances (TBARS) produced. Briefly, the samples were diluted by buffered saline $(1: 5)$ and $800 \mu \mathrm{l}$ of trichloroacetic acid (TCA, $28 \% \mathrm{w} / \mathrm{v}$ ) was added to $400 \mu \mathrm{l}$ of this mixture and centrifuged in $3000 \times \mathrm{g}$ for $30 \mathrm{~min}$. Then, the precipitation was dissolved in sulfuric acid and $600 \mu \mathrm{l}$ of the mixture was added to $150 \mu \mathrm{l}$ of TBA $(1 \% \mathrm{w} / \mathrm{v})$. The mixture was then incubated for $15 \mathrm{~min}$ in a boiling water bath. Following incubation, $4 \mathrm{ml}$ of $n$-butanol was added, the solution was centrifuged, cooled and the absorption of the supernatant was recorded at $532 \mathrm{~nm}$ using a UV-160-A Shimadzu double beam spectrophotometer (Japan). The calibration curve of a 1,1,3,3-tetraethoxypropan standard solution was used to determine the concentrations of TBA-MDA adducts in samples (41).

\section{Statistical Analysis}

The statistical analysis was performed with the use of StatsDirect version 2.6.2. Continuous data are expressed as mean \pm SE. Statistical analysis was initially performed by Kolmogorov-Smirnov normality test. Unpaired twosample student's $t$-test was used for analysis of continuous data and Chi-square test was used for categorical data if applicable. A $P$-value $<0.05$ was considered to be statistically significant.

\section{Results}

\section{Patients' Characteristics}

Twenty-one patients with type 2 diabetes mellitus (12 male and 9 female) who fulfilled the enrolment criteria entered into the study between July 2006 and January 2007. Eleven patients were randomized to receive S. khuzestanica and 10 to receive placebo. No patients were withdrawn from the study. Demographic characteristics did not differ significantly between two groups (Table 1). No adverse events were reported from the subjects throughout the study period. 


\section{Patients' Blood Glucose and Lipid Profile and Oxidative Stress Biomarkers}

Table 2 shows changes found on metabolic parameter values in both groups between baseline and end of therapy. Patients in the $S$. khuzestanica group showed significant decrease in total cholesterol $(P=0.008)$, LDL$\mathrm{C}(P=0.03)$ and increase in HDL-C $(P=0.02)$ and TAP $(P=0.007)$ measured from baseline to 2 months while patients in the placebo group showed no changes. The other parameters including blood glucose, triglyceride, creatinin and TBARS were not significantly altered in S. khuzestanica group.

\section{Discussion}

Recently, there has been a considerable interest in finding natural antioxidants from plant materials to replace synthetic ones. Data from both scientific reports and

Table 1. Baseline characteristics of subjects

\begin{tabular}{lll}
\hline & $\begin{array}{l}\text { Satureja khuzestanica } \\
(n=11)\end{array}$ & $\begin{array}{l}\text { Placebo } \\
(n=10)\end{array}$ \\
\hline Age (year) & $50.65 \pm 2.11$ & $51.71 \pm 2.05$ \\
Male/female $(n)$ & $6 / 5$ & $6 / 4$ \\
SBP $(\mathrm{mmHg})$ & $128.60 \pm 3.67$ & $127.58 \pm 3.71$ \\
DBP $(\mathrm{mmHg})$ & $80.70 \pm 3.03$ & $81.08 \pm 3.60$ \\
BMI $\left(\mathrm{kg} \mathrm{m}^{-2}\right)$ & $26.35 \pm 1.70$ & $27.63 \pm 2.44$ \\
$\mathrm{HbA}_{1 \mathrm{c}}(\%)$ & $7.25 \pm 1.42$ & $7.65 \pm 1.50$ \\
Concomitant therapy & & \\
$\quad$ Glibenclamide $(n)$ & 8 & 6 \\
$\quad$ Metformin $(n)$ & 5 & 5 \\
Statins $(n)$ & 9 & 9 \\
fibrates $(n)$ & 2 & 1 \\
\hline
\end{tabular}

SBP, systolic blood pressure; DBP, diastolic blood pressure; BMI, body mass index; $\mathrm{HbA}_{1 \mathrm{c}}$, glycated hemoglobin; $n$, number of patients.

The values are expressed as mean $\pm \mathrm{SE}$. laboratory studies show that plants contain a large variety of substances that possess antioxidant activity (42). Phytochemicals with antioxidant effects include some cinnamic acids, coumarins, diterpenes, flavonoids, lignans, monoterpenes, phenylpropanoids, tannins and triterpenes (43). Therefore, it seems that plants particularly those with high levels and strong antioxidant compounds have an important role in improvement of disorders involving oxidative stress such as diabetes mellitus (37). S. khuzestanica, an endemic plant of Iran, possess antioxidant properties $(23,26)$ and is expected to be useful in diabetes and its complications.

The results of this study, show the significant effects of S. khuzestanica in improving patients' total cholesterol, LDL-C, HDL-C and TAP. In previous study, it was revealed that $S$. khuzestanica significantly decrease fasting blood glucose and triglyceride levels in diabetic and hyperlipidemic rats. It also improved the lipid peroxidation and TAP levels (23). In another investigation, it was reported that $S$. khuzestanica does not affect blood glucose concentrations of rats (24). The main constituents of $S$. khuzestanica are isopropanoids such as carvacrol, thymol and flavonoids. It has been shown that thymol and carvacrol significantly decrease the serum cholesterol levels. They increased microsomal geranyl pyrophosphate pyrophosphatase activity by 2-fold. The structural diversity of the isopropanoids which suppress cholesterol synthesis may be reconciled by their ability to increase pyrophosphatase activity, thus leading to the production of the endogenous, post-transcriptional regulator of 3-hydroxy-3-methylglutaryl coenzyme A reductase activity (44). Flavonoids have also shown antioxidant and anti hyperlipidemic properties. Soy isoflavones significantly reduced serum total and LDL-C but did not change HDL-C and triacylglyceride (45). Covas et al. (46) reported that consumption of polyphenolrich olive oil increases HDL-C levels and lowers levels of oxidative stress markers and oxidized LDL-C.

Table 2. Levels of blood glucose, triglyceride, total cholesterol, LDL-C, HDL-C, creatinine, TAP, and lipid peroxidation before and after administration of Satureja Khuzestanica or placebo

\begin{tabular}{|c|c|c|c|c|c|c|}
\hline \multirow[b]{2}{*}{ Parameters } & \multicolumn{3}{|c|}{ Satureja khuzestanica $(n=11)$} & \multicolumn{3}{|c|}{ Placebo $(n=10)$} \\
\hline & Before & After & $P$-value & Before & After & $P$-value \\
\hline Glucose $\left(\mathrm{mg} \mathrm{dl}^{-1}\right)$ & $163 \pm 10.4$ & $160 \pm 8.9$ & 0.7 & $165.9 \pm 17$ & $201 \pm 12$ & 0.37 \\
\hline Total cholesterol $\left(\mathrm{mg} \mathrm{dl}^{-1}\right)$ & $202.8 \pm 3.5$ & $156.2 \pm 4.2$ & 0.008 & $189.1 \pm 6.5$ & $214 \pm 9$ & 0.1 \\
\hline LDL-C $\left(\mathrm{mg} \mathrm{dl}^{-1}\right)$ & $120 \pm 4.5$ & $103 \pm 3.9$ & 0.03 & $123 \pm 6$ & $122 \pm 6.6$ & 0.8 \\
\hline HDL-C $\left(\mathrm{mg} \mathrm{dl}^{-1}\right)$ & $45.3 \pm 3.2$ & $50 \pm 1.6$ & 0.02 & $45 \pm 2$ & $44.4 \pm 3.2$ & 0.3 \\
\hline TAP $\left(\mathrm{mmoll}^{-1}\right)$ & $336 \pm 48$ & $477 \pm 24$ & 0.007 & $381 \pm 0.45$ & $417 \pm 15.8$ & 0.1 \\
\hline TBARS $\left(\mu \mathrm{moll} 1^{-1}\right)$ & $152 \pm 17.3$ & $164 \pm 19.1$ & 0.51 & $151 \pm 12.5$ & $164 \pm 19.1$ & 0.1 \\
\hline
\end{tabular}

HDL-C, high-density lipoprotein-cholesterol; LDL-C, low-density lipoprotein-cholesterol; TAP, total antioxidant power; TBARS, thiobarbituric acid reactive substances.

The values are expressed as mean $\pm \mathrm{SE}$. 
Recently, S. khuzestanica has been found to prevent from malathion-induced toxicity through improvement of oxidative stress parameters (47). Since free radicals has a pivotal role in pathogenesis and complications of diabetes (37), every natural or synthetic compound that can reduce oxidative stress may be beneficial in the management of diabetes (48-53). One of our expectations in the present study was to observe significant reductions in blood glucose and level of lipid peroxidation by S. khuzestanica treatment but this did not happen. The first thing that comes to our mind is that dose of S. khuzestanica was low and duration of the study was small. On the other hand, disturbed lipid profile is usually observed in diabetic patients and most of diabetics usually receive lipid lowering agents mostly statins that accompany with some adverse effects and also costs a lot for diabetic patients $(54,55)$. Introduction of new herbal medicines like S. khuzestanica as a drug with low price and safe can be helpful.

\section{Conclusion}

In summary, it seems that $S$. khuzestanica have a lipid lowering and antioxidant properties in human and since it has not shown any adverse effects, it could be used as a supplement in diabetic patients with hyperlipidemia. The exact role of this herbal supplement in management of hyperlipidemic type- 2 diabetic patients remains to be elucidated by larger sample size and longer term clinical trials.

\section{Acknowledgements}

This study was supported by a grant from EMRC and PSRC of TUMS.

\section{References}

1. Jamzad Z. A new species of the genus Satureja (Labiatae) from Iran. Iran J Bot 1996;6:215-8.

2. Zargari A. Medicinal Plants. Tehran: Tehran University Publications, 1990, 42.

3. Sahin F, Karaman I, Gulluce M, Ogutcu H, Sengul M, Adiguzel A, et al. Evaluation of antimicrobial activities of Satureja hortensis L. $J$ Ethnopharmacol 2003;87:61-5.

4. Skocibusic M, Bezic N. Phytochemical analysis and in vitro antimicrobial activity of two Satureja species essential oils. Phytother Res 2004;18:967-70.

5. Sonboli A, Fakhari A, Kanani MR, Yousefzadi M. Antimicrobial activity, essential oil composition and micromorphology of trichomes of Satureja laxiflora C. Koch from Iran. Z Naturforsch [C] 2004;59:777-81.

6. Yamasaki K, Nakano M, Kawahata T, Mori H, Otake T, Ueba N, et al. Anti-HIV-1 activity of herbs in Labiatae. Biol Pharm Bull 1998;21:829-33.

7. Abad MJ, Bermejo P, Gonzales E, Iglesias I, Irurzun A, Carrasco L. Antiviral activity of Bolivian plant extracts. Gen Pharmacol 1999;32:499-503.

8. Radonic A, Milos M. Chemical composition and in vitro evaluation of antioxidant effect of free volatile compounds from Satureja montana L. Free Radic Res 2003;37:673-9.
9. Cetojevic-Simin DD, Canadanovic-Brunet JM, Bogdanovic GM, Cetkovic GS, Tumbas VT, Djilas SM. Antioxidative and antiproliferative effects of Satureja montana L. extracts. $J$ BUON 2004;9:443-9.

10. Mosaffa F, Behravan J, Karimi G, Iranshahi M. Antigenotoxic effects of Satureja hortensis L. on rat lymphocytes exposed to oxidative stress. Arch Pharm Res 2006;29:159-64.

11. Lampronti I, Saab AM, Gambari R. Antiproliferative activity of essential oils derived from plants belonging to the Magnoliophyta division. Int $J$ Oncol 2006;29:989-95.

12. Sulsen V, Guida C, Coussio J, Paveto C, Muschietti L, Martino V. In vitro evaluation of trypanocidal activity in plants used in Argentine traditional medicine. Parasitol Res 2006;98:370-4.

13. van Baren C, Anao I, Leo Di Lira P, Debenedetti S, Houghton P, Croft $S$, et al. Triterpenic acids and flavonoids from Satureja parvifolia. Evaluation of their antiprotozoal activity. Z Naturforsch [C] 2006;61:189-92.

14. Tampieri MP, Galuppi R, Macchioni F, Carelle MS, Falcioni L, Cioni PL, et al. The inhibition of Candida albicans by selected essential oils and their major components. Mycopathologia 2005;159:339-45.

15. Boyraz N, Ozcan M. Inhibition of phytopathogenic fungi by essential oil, hydrosol, ground material and extract of summer savory (Satureja hortensis L.) growing wild in Turkey. Int $J$ Food Microbiol 2005;107:238-42.

16. Hajhashemi V, Ghannadi A, Pezeshkian SK. Antinociceptive and anti-inflammatory effects of Satureja hortensis L. extracts and essential oil. J Ethnopharmacol 2002;82:83-7.

17. Suarez A, Echandi MM, Ulate G, Ciccio JF. Pharmacological activity of the essential oil of Satureja viminea (Lamiaceae). Rev Biol Trop 2003;51:247-52.

18. Hajhashemi V, Sadraei H, Ghannadi AR, Mohseni M. Antispasmodic and anti-diarrhoeal effect of Satureja hortensis L. essential oil. J Ethnopharmacol 2000;71:187-92.

19. Sanchez de Rojas VR, Somoza B, Ortega T, Villar AM. Isolation of vasodilatory active flavonoids from the traditional remedy Satureja obovata. Planta Med 1996;62:272-4.

20. Ramon Sanchez de Rojas V, Somoza B, Ortega T, Villar AM, Tejerina T. Vasodilatory effect in rat aorta of eriodictyol obtained from Satureja obovata. Planta Med 1999;65:234-8.

21. Amanlou M, Dadkhah F, Salehnia A, Farsam H, Dehpour AR. An anti-inflammatory and anti-nociceptive effects of hydroalcoholic extract of Satureja khuzistanica Jamzad extract. J Pharm Pharm Sci 2005;8:102-6.

22. Amanlou M, Fazeli MR, Arvin A, Amin HG, Farsam H. Antimicrobial activity of crude methanolic extract of Satureja khuzistanica. Fitoterapia 2004;75:768-70.

23. Abdollahi M, Salehnia A, Mortazavi SH, Ebrahimi M, Shafiee A, Fouladian F, et al. Antioxidant, antidiabetic, antihyperlipidemic, reproduction stimulatory properties and safety of essential oil of Satureja khuzestanica in rat in vivo: a oxicopharmacological study. Med Sci Monit 2003;9:331-5.

24. Saadat M, Pournourmohammadi S, Donyavi M, Khorasani R, Amin G, Nazar Salehnia A, et al. Alteration of rat hepatic glycogen phosphorylase and phosphoenolpyruvate carboxykinase activities by Satureja khuzestanica Jamzad essential oil. J Pharm Pharm Sci 2004;7:310-4.

25. Haeri S, Minaie B, Amin G, Nikfar S, Khorasani R, Esmaily H, et al. Effect of Satureja khuzestanica essential oil on male rat fertility. Fitoterapia 2006;77:495-9.

26. Ghazanfari G, Minaie B, Yasa N, Ashtaral Nakhai L, Mohammadirad A, Nikfar S, et al. Biochemical and histopathological evidences for beneficial effects of Satureja khuzestanica Jamzad essential oil on the mouse model of inflammatory bowel disease. Toxicol Mech Methods 2006;16:365-72.

27. El-Gazzar A, Watson L. A taxonomic study of Labiatae and related genera. New Phytol 1970;69:451-86.

28. Farsam H, Amanlou M, Radpour MR, Salehinia AN, Shafiee A. Composition of the essential oils of wild and cultivated Satureuja khuzistanica Jamzad from Iran. Flavour Frag $J$ 2004;19:308-10.

29. Moghaddam FM, Farimani MM, Salahvarzi S, Amin G. Chemical constituents of dichloromethane extract of cultivated Satureja khuzistanica. Evid Based Complement Alternat Med 2007;4:95-8. 
30. Burits M, Bucar F. Antioxidant activity of Nigella sativa essential oil. Phytother Res 2000;14:323-8.

31. Lambert RJ, Skandamis PN, Coote PJ, Nychas GJ. A study of the minimum inhibitory concentration and mode of action of oregano essential oil, thymol and carvacrol. $J$ Appl Microbiol 2001;91:453-62.

32. Heim KE, Tagliaferro AR, Bobilya DJ. Flavonoid antioxidants: chemistry, metabolism and structure-activity relationships. $J$ Nutr Biochem 2002;13:572-84.

33. Vardar-Unlu G, Candan F, Sokmen A, Donmez E, Tepe B. Antimicrobial and antioxidant activity of the essential oil and methanol extracts of Thymus pectinatus Fisch. et Mey. Var. pectinatus (Lamiaceae). J Agric Food Chem 2003;51:63-7.

34. Brown DJ, Goodman J. A review of vitamins A, C, and E and their relationship to cardiovascular disease. Clin Excell Nurse Pract 1998;2:10-22.

35. Hasanain B, Mooradian AD. Antioxidant vitamins and their influence in diabetes mellitus. Curr Diab Rep 2002;2:448-56.

36. Andreassi MG. Coronary atherosclerosis and somatic mutations: an overview of the contributive factors for oxidative DNA damage. Mutat Res 2003;543:67-86.

37. Rahimi R, Nikfar S, Larijani B, Abdollahi M. A review on the role of antioxidants in the management of diabetes and its complications. Biomed Pharmacother 2005;59:365-73.

38. American Diabetes Association. Standards of medical care in diabetes-2006. Diab care 2006;29:S4-2.

39. Third Report of the National Cholesterol Education Program (NCEP). Expert Panel on Detection, Evaluation, and Treatment of High Blood Cholesterol in Adults (Adult Treatment Panel III) final report. Circulation 2002;106:3143-421.

40. Benzi IF, Strain S. Ferric reducing antioxidant assay. Meth Enzymol 1999;292:15-27.

41. Satho K. Serum lipid peroxidation in cerebrovascular disorders determined by a new colorimetric method. Clin Chim Acta 1978;90:37-43.

42. Chanwitheesuk A, Teerawutgulrag A, Rakariyatham N. Screening of antioxidant activity and antioxidant compounds of some edible plants of Thailand. Food Chem 2005:92:491-7.

43. Larkins N, Wynn S. Pharmacognosy: phytomedicines and their mechanisms. Vet Clin North Am Small Anim Pract 2004:34:291-327.

44. Case GL, He L, Mo H, Elson CE. Induction of geranyl pyrophosphate pyrophosphatase activity by cholesterol-suppressive isoprenoids. Lipids 1995;30:357-9.

45. Taku K, Umegaki K, Sato Y, Taki Y, Endoh K, Watanabe S. Soy isoflavones lower serum total and LDL cholesterol in humans: a meta-analysis of 11 randomized controlled trials. Am J Clin Nutr 2007;85:1148-56.
46. Covas MI, Nyyssonen K, Poulsen HE, Kaikkonen J, Zunft HJ, Kiesewetter $\mathrm{H}$, et al. EUROLIVE Study Group. The effect of polyphenols in olive oil on heart disease risk factors: a randomized trial. Ann Intern Med 2006;145:333-41.

47. Basiri S, Esmaily H, Vosough-Ghanbari S, Mohammadirad A, Yasa N, Abdollahi M. Improvement by Satureja khuzestanica essential oil of malathion-induced red blood cells acetylcholinesterase inhibition and altered hepatic mitochondrial glycogen phosphorylase and phosphoenolpyruvate carboxykinase activities. Pestic Biochem Physiol 2007:89:124-9.

48. Astaneie F, Afshari M, Mojtahedi A, Mostafalou S, Zamani MJ, Larijani B, et al. Total antioxidant capacity and levels of epidermal growth factor and nitric oxide in blood and saliva of insulindependent diabetic patients. Arch Med Res 2005;36:376-81.

49. Sarkhail P, Rahmanipour S, Fadyevatan S, Mohammadirad A, Dehghan G, Amin G, et al. Antidiabetic effect of Phlomis anisodonta: effects on hepatic cells lipid peroxidation and antioxidant enzymes in experimental diabetes. Pharmacol Res 2007;56:261-6.

50. Larijani B, Afshari M, Astanehi-Asghari F, Mojtahedi A, Rezaie A, Hosseinnezhad A, et al. Effect of short-term carvedilol therapy on salivary and plasma oxidative stress parameters and plasma glucose level in type II diabetes. Therapy 2006;3:119-23.

51. Milani E, Nikfar S, Khorasani K, Zamani MJ, Abdollahi M. Reduction of diabetes-induced oxidative stress by phosphodiesterase inhibitors in rats. Comp Biochem Physiol C Toxicol Pharmacol 2005; 140:251-5.

52. Radfar M, Larijani B, Hadjibabaie M, Rajabipour B, Mojtahedi A, Abdollahi M. Effects of pentoxifylline on oxidative stress and levels of EGF and NO in blood of diabetic type-2 patients; a randomized, double-blind placebo-controlled clinical trial. Biomed Pharmacother 2005;59:302-6.

53. Afshari M, Larijani B, Rezaie A, Mojtahedi A, Zamani MJ, Astanehi-Asghari F, et al. Ineffectiveness of allopurinol in reduction of oxidative stress in diabetic patients; a randomized, doubleblind placebo-controlled clinical trial. Biomed Pharmacother 2004:58:546-50.

54. Hadjibabaie M, Vosough-Ghanbari S, Radfar M, Gholami K, Khoei SH, Nakhjavani M, et al. Comparative efficacy of daily versus alternate-day dosing of atorvastatin in Type 2 diabetic patients. Therapy 2007:4:541-5.

55. Hadjibabaie M, Gholami K, Khalili H, Khoei SH, Nakhjavani M, Rayati K, et al. Comparative efficacy and safety of atorvastatin, simvastatin, and lovastatin in the management of dyslipidemic type2 diabetic patients. Therapy 2006;3:759-64.

Received July 2, 2007; accepted February 14, 2008 


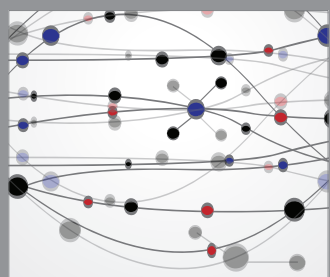

The Scientific World Journal
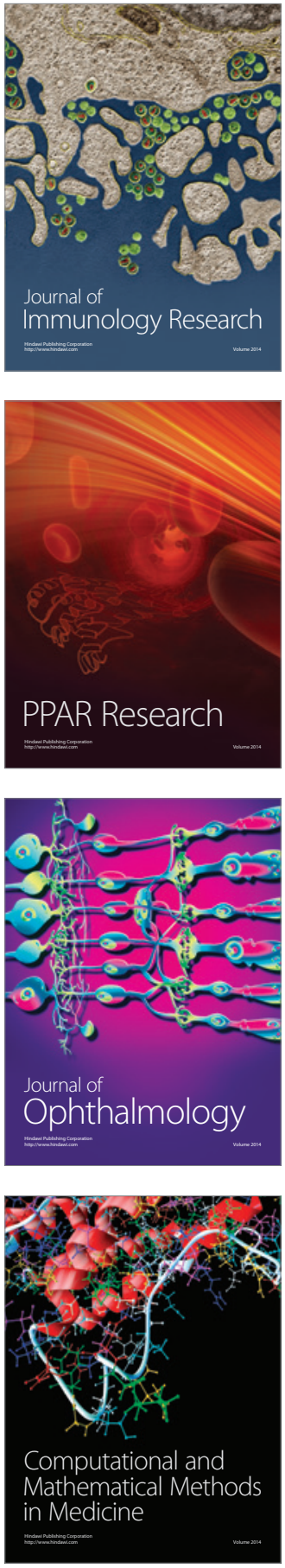

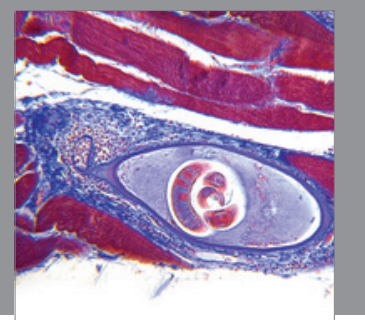

Gastroenterology

Research and Practice
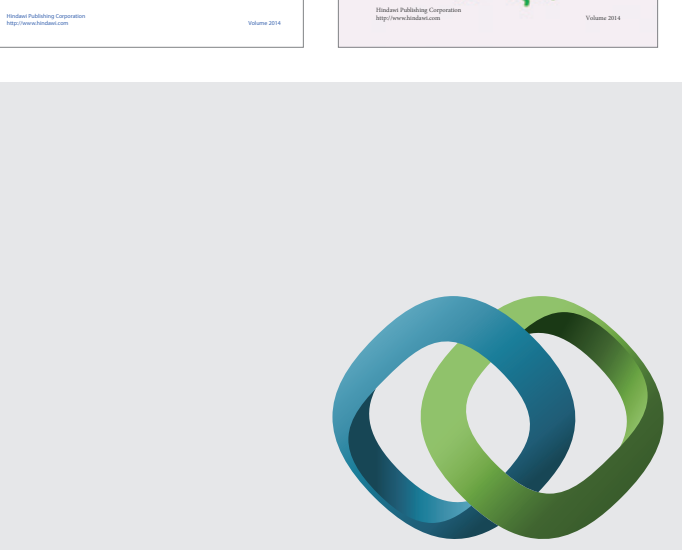

\section{Hindawi}

Submit your manuscripts at

http://www.hindawi.com
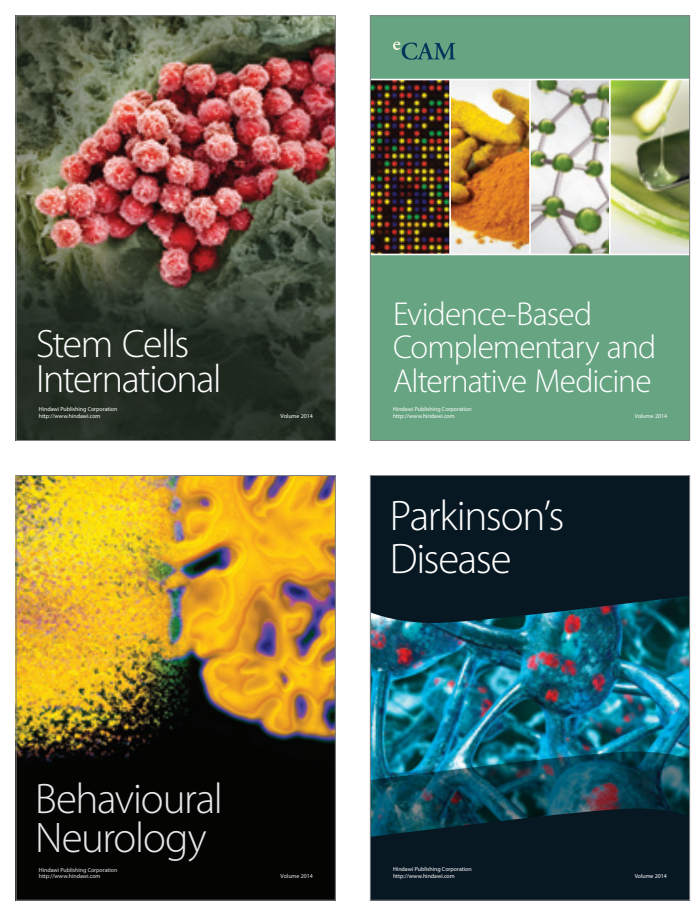

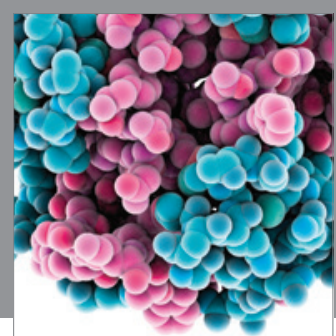

Journal of
Diabetes Research

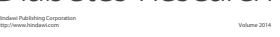

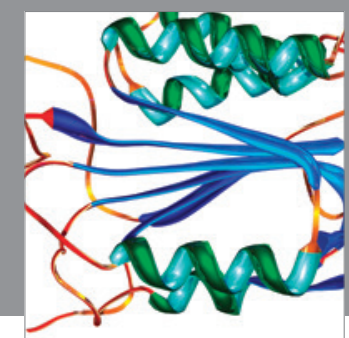

Disease Markers
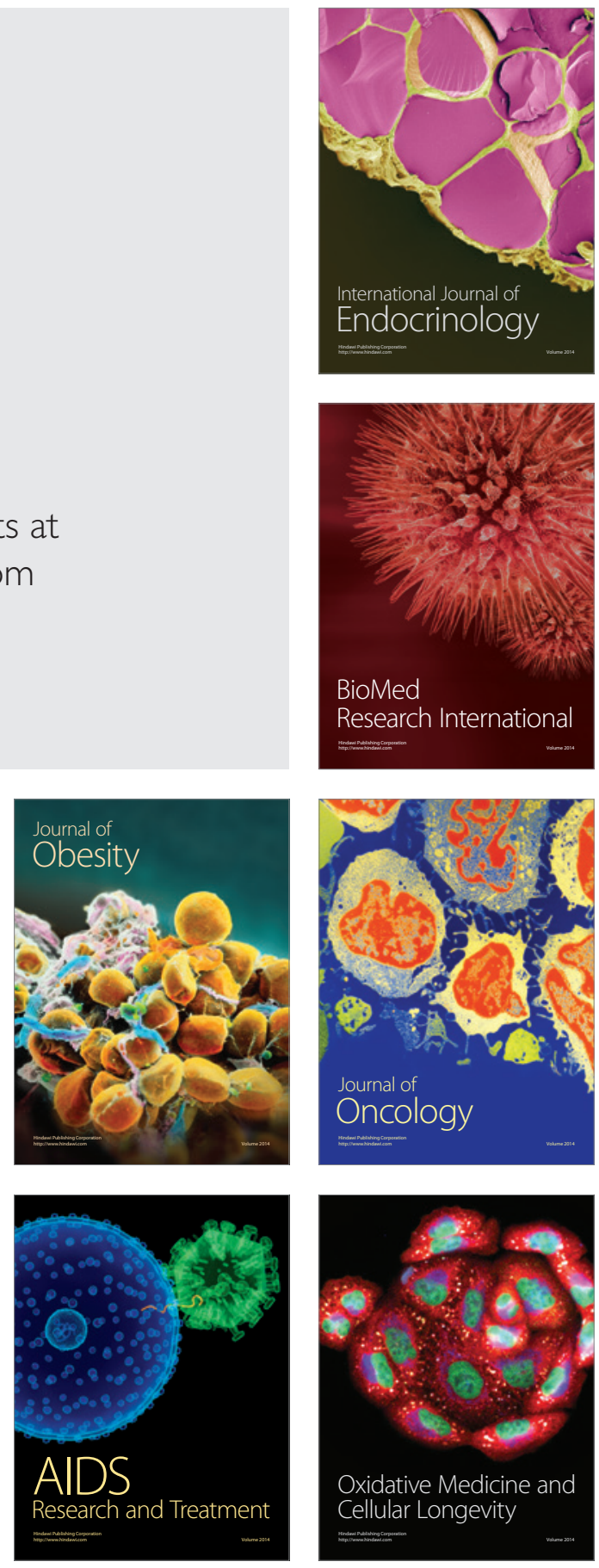